\title{
The therapeutic effect of interleukin-22 in high androgen-induced polycystic ovary syndrome
}

\author{
Xinyu Qi1,3, Chuyu Yun2, Baoying Liao 1,3,4, Jie Qiao1,3,4,5,6 and Yanli Pang1,3,4 \\ ${ }^{1}$ Center for Reproductive Medicine, Department of Obstetrics and Gynecology, Peking University Third Hospital, Beijing, China \\ ${ }^{2}$ Key Laboratory of Molecular Cardiovascular Science, Ministry of Education, School of Basic Medical Sciences, Peking University, Beijing, China \\ ${ }^{3}$ Key Laboratory of Assisted Reproduction (Peking University), Ministry of Education, Beijing, China \\ 4Beijing Key Laboratory of Reproductive Endocrinology and Assisted Reproductive Technology, Beijing, China \\ ${ }^{5}$ National Clinical Research Center for Obstetrics and Gynecology, Beijing, China \\ ${ }^{6}$ Research Units of Comprehensive Diagnosis and Treatment of Oocyte Maturation Arrest, Beijing, China
}

Correspondence should be addressed to Y Pang: yanlipang@bjmu.edu.cn

\begin{abstract}
Polycystic ovary syndrome (PCOS) is a complex syndrome involving both endocrine and metabolic disorders. Gut microbiota and the intestinal immune factor IL-22 play an important role in the pathogenesis of PCOS. However, the therapeutic role of IL-22 in high androgen-induced PCOS mice is not clear. We aimed to determine the therapeutic effects of IL-22 on the DHEA-induced PCOS mouse model and to explore the possible mechanism of IL-22 in regulating hyperandrogenism-associated PCOS. Insulin resistance levels and ovarian functions were investigated in DHEA-induced PCOS mice with or without additional IL-22 treatment. We found that IL-22 could reverse insulin resistance, disturbed estrous cycle, abnormal ovary morphology, and decreased embryo number in DHEA mice. Mechanistically, IL-22 upregulated the browning of white adipose tissue in DHEA mice. This study demonstrated that IL-22-associated browning of white adipose tissue regulated insulin sensitivity and ovarian functions in PCOS, suggesting that IL-22 may be of value for the treatment of PCOS with a hyperandrogenism phenotype.
\end{abstract}

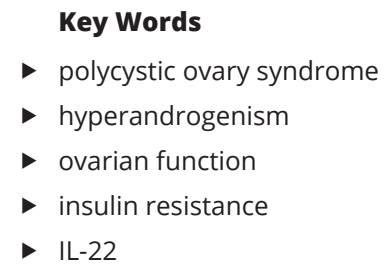

Journal of Endocrinology (2020) 245, 281-289

\section{Introduction}

Polycystic ovary syndrome (PCOS) is one of the most common endocrine and metabolic disorders in women of reproductive age. The prevalence of PCOS is approximately $5-10 \%$ (Norman et al. 2007). It is characterized by a combination of signs and symptoms of androgen excess (hirsutism and/or hyperandrogenemia) and ovarian dysfunction (anovulation, oligoovulation and/or polycystic ovarian morphology) and is frequently associated with abdominal adiposity, insulin resistance, obesity, metabolic disorders and cardiovascular risk factors (Dumesic et al. 2015). Due to the complex etiology and its heterogeneous nature, the mechanism of ovulatory dysfunction and insulin resistance in women with PCOS remains elusive.
The cytokine interleukin-22 (IL-22), which is produced by intestinal group 3 innate lymphoid cells (ILC3), plays an important role in the regulation and function of host defense and inflammatory diseases. It helps to shape and constrain the commensal community in various organs, including the intestine, the lung, and the stomach (Ouyang \& O'Garra 2019). In tissues and organs with high IL-22 receptor expression, IL-22 helps to prevent cell death and tissue damage caused by inflammation and infection, therefore promoting wound healing and restoring tissue integrity and homeostasis (Rutz et al. 2014). To date, IL-22 has been used in some preclinical inflammatory models, such as hepatitis, pancreatitis, wound healing, graft vs 
host disease (GVHD), and inflammatory bowel disease (IBD). Clinical trials have shown that the administration of exogenous IL-22 could provide therapeutic benefits (Wang et al. 2019).

Recently, our group reported that the IL-22 levels in serum and follicle fluid of PCOS patients were decreased and that IL-22 administration could help to improve insulin resistance, ovarian dysfunction, and infertility in intestinal bacteria or prenatal anti-Müllerian hormone induced PCOS like mice (Qi et al. 2019). However, the role of IL-22 in hyperandrogenism-associated PCOS is unknown. DHEA-induced mice could better represent the hyperandrogenic phenotype in PCOS patients. In the current study, a DHEA model was used to further validate the therapeutic role of IL-22 in different subtypes of PCOS.

\section{Materials and methods}

\section{Animal model}

Female prepuberal (21 days old) mice of the C57BL/ 6 strain (Department of Experimental Animal Sciences, Peking University Health Science Center, Beijing, China) were randomly divided into the control group, DHEA group, and DHEA+IL-22 group and housed three per cage. All animals were maintained under controlled temperature and lighting conditions (12 h light:12 h darkness cycle) with free access to food and water. The animal experimental procedures were approved by the Animal Care and Use Committee of Peking University Health Science Center according to the national legislation for animal care. Mice in the DHEA group and the DHEA+IL-22 group were injected daily with DHEA (Sigma, D4000) (6 mg/100 g body weight, dissolved in $0.1 \mathrm{~mL}$ sesame oil) subcutaneously for 21 days and PBS or IL-22 (100 $\mu \mathrm{g} / \mathrm{kg} / \mathrm{d}, \mathrm{R} \& \mathrm{D})$ intraperitoneally for another 21 days. The control group were injected daily with sesame oil for 21 days and PBS for another 21 days. Half of the mice from each group were used for implantation assessment and the other half were killed for sample collection. The control and DHEA+IL-22 mice were killed at diestrus except for the DHEA mice because they had a disturbed estrous cycle. They were killed by decapitation after a 12-h fasting and an anesthesia with diethyl ether at $4^{\circ} \mathrm{C}$. The serum was collected for sex hormone and IL-22 detection. The ovaries and part of s.c. adipose tissues from the inguinal region were processed for staining with hematoxylin and eosin (H\&E). The brown fat tissues were collected from the interscapular region. The remaining s.c. adipose tissues and brown adipose tissues were quickly frozen and stored at $-80^{\circ} \mathrm{C}$.

\section{Vaginal smears and estrus cycle determination}

From the 31st day to the 40th day, after the 1st day of treatment, vaginal smears were taken daily at 10:00 h and the specimens were stained according to Shore's staining. The stage of the estrus cycle was determined by the predominant cell type in vaginal smears.

\section{Glucose tolerance test and insulin tolerance test}

Before the GTT experiment, mice were fasted for $12 \mathrm{~h}$. Glucose levels were measured by tail vein blood sampling using a blood glucose ACCU-CHEK Performa (Roche Diagnostics). After fasting, the glucose levels were measured, and the mice were intraperitoneally injected with D-glucose (Sigma) ( $2 \mathrm{~g} / \mathrm{kg}$ body weight), and tail sampling was performed at 15-, 30-, 60-, 90-, and 120-min post-intraperitoneal injection.

Two days after the GTT experiment, the mice were fasted for $4 \mathrm{~h}$ before the ITT experiment. Glucose levels were measured by tail vein blood sampling using a blood glucometer. After fasting, the glucose levels were measured, and the mice were intraperitoneally injected with insulin (Wanbang Biopharmaceuticals, Xuzhou, China) (1 IU/kg body weight), and tail sampling was performed at 15-, 30-, 60-, 90-, and 120-min post-intraperitoneal injection.

\section{Serum analysis}

The blood samples were centrifuged at $4^{\circ} \mathrm{C}$ for $15 \mathrm{~min}$ at 956 $\boldsymbol{g}$ and stored at $-80^{\circ} \mathrm{C}$ for subsequent serum determinations. The levels of testosterone (S10940093, range: 0.1-20 $\mathrm{ng} / \mathrm{mL}$, sensitivity: $0.02 \mathrm{ng} / \mathrm{mL}$ ), luteinizing hormone (LH, S10950161, range: 5-200 mIU/mL, sensitivity: $\leq 1.0$ $\mathrm{mIU} / \mathrm{mL}$ ), 17ß-estradiol (20172400011, range: 5-4000 $\mathrm{pg} / \mathrm{mL}$, sensitivity: $\leq 2 \mathrm{pg} / \mathrm{mL}$ ), follicle-stimulating hormone (FSH, S10950154, range: $2.5-100 \mathrm{mIU} / \mathrm{mL}$, sensitivity: $<1.0$ $\mathrm{mIU} / \mathrm{mL}$ ), and insulin (S10930046, range: $5-405 \mu \mathrm{IU} / \mathrm{mL}$, sensitivity: $2 \mu \mathrm{IU} / \mathrm{mL}$ ) were determined with ${ }^{125}$ I-labelled RIA kits (Beijing North Institute of Biological Technology and Beijing Kemei Diagnose, Beijing, China). Homeostatic model assessment of IR (HOMA-IR) levels were defined as $\mathrm{HOMA}=$ insulin $\quad(\mathrm{mUI} / \mathrm{mL}) \times$ glucose $\quad(\mathrm{mmol} / \mathrm{L}) / 22.5$ (Antunes et al. 2016). IL-22 levels were determined by ELISA kits for mice (R\&D, M2200).

\section{Implantation assessment}

To examine implantation, female mice were mated with proven stud males. The next day, successful mating was judged by observation of a vaginal plug. After 10 days, 
female mice were killed and examined at implantation sites to confirm pregnancy.

\section{Morphology and immunohistochemistry}

The ovaries and adipose tissues were quickly collected and fixed in 4\% paraformaldehyde, placed in 70\% ethanol, and dehydrated and embedded with paraffin. The sections were prepared and stained with H\&E (Beisuo Biotech Company, Beijing, China). The ovaries were longitudinally and serially sectioned at $5 \mu \mathrm{m}$, and every fifth section was mounted on a glass slide and observed under light microscopy for histomorphology examinations. The number of corpora lutea and cystic follicles of the ovaries was counted. The results were confirmed by a pathologist.

Immunohistochemistry was performed using a rabbit anti-UCP1 antibody (1:100 dilution; Abcam, ab10983) for $60 \mathrm{~min}$ at $37^{\circ} \mathrm{C}$. After washing with PBS, the sections were sequentially incubated with a goat anti-rabbit secondary antibody (PV-6001; Zhongshan Goldenbridge Biotechnology, Beijing, China) for $30 \mathrm{~min}$ at room temperature. Diaminobezidin (DAB) was added to change the color by incubating for 2-5 min, and counterstaining was performed with hematoxylin for $5 \mathrm{~min}$, after which the slides were dehydrated with an increasing ethanol concentration and xylene and then sealed.

\section{Quantitative real-time PCR analysis}

Real-time qPCR analysis was performed using SYBR Green PCR master mix (Invitrogen), and expression levels were assessed by the ABI 7500 real-time PCR system (Applied Biosystems). In order to exclude the DNA contamination in mRNAs, we designed one-to-one primers to ensure the specificity of the primers. High temperature sterilization or UV irradiation were used to destroy the nucleic acid in the tips, EP tubes, and environment before adding samples to exclude DNA contamination. All qPCRs were carried out in a final volume of $20 \mu \mathrm{L}$ following the manufacturer's instructions (Invitrogen). The amplification thermal cycling conditions were $95^{\circ} \mathrm{C}$ for $2 \mathrm{~min}$, followed by 40 cycles at $95^{\circ} \mathrm{C}$ for $15 \mathrm{~s}$ and $60^{\circ} \mathrm{C}$ for $40 \mathrm{~s}$. Relative mRNA expression was determined by the $2^{-\Delta \Delta C T}$ method normalized to $A c t b$ mRNA levels. The qPCR primers used in this study can be found in Supplementary Table 1 (see section on supplementary materials given at the end of this article).

\section{Statistics}

The sample distribution was determined by the Kolmogorov-Smirnov normality test. No data were excluded from the data analysis. One-way ANOVA followed by Tukey's post-hoc test was used to evaluate the statistical significance of differences among three groups. For the nonparametric tests, Kruskal-Wallis test was used to analyze differences among three experimental groups, followed by Dunn's post hoc analysis. Data are shown as means \pm s.E.M. or as medians with interquartile ranges. $P<0.05$ was considered statistically significant. All analyses were conducted with SPSS version 19.0.

\section{Results}

\section{IL-22 treatment reverses metabolic dysfunction in DHEA induced PCOS mice}

To verify the treatment effect of IL-22 administration in hyperandrogenism-associated PCOS, IL-22 was injected into DHEA-treated mice (Fig. 1A). The glucose metabolism was detected in control, DHEA, and DHEA + IL-22 groups. In the glucose tolerance test (GTT), after administration of glucose, we found that IL-22 resulted in markedly lower glucose levels in the DHEA+IL-22 group than the levels in the DHEA group at the 15-, 30-, 60-, 90-, and 120-min points, indicating that IL-22 could restore the impaired glucose tolerance of DHEA mice to normal (Fig. 1B). Similar to the results of GTT, in the insulin tolerance test (ITT), after injection with insulin, the glucose levels in the DHEA+IL-22 group were significantly downregulated at the 15-, 30-, 60-, and 120-min points compared with the levels in the DHEA group (Fig. 1C). Statistically, the DHEA+IL-22 group showed a lower GTT area under the curve (GTT AUC) and ITT area under the curve (ITT AUC) compared with those of the DHEA group (Fig. 1D and E). We also found that administration of IL-22 to the PCOS mice decreased fasting glucose levels, fasting insulin levels, and HOMA-IR (Fig. 1F, G and H). Based on these results, we found that administration of IL-22 to PCOS mice reversed insulin resistance similar to a normal level.

\section{IL-22 treatment improved hormone disorders in DHEA-treated mice}

Hormone levels are important features of ovarian function and are closely related to the severity of PCOS. IL-22 administration significantly decreased the serum testosterone levels in DHEA-treated mice to similar levels as the control group (Fig. 2A), whereas the estradiol levels among the three groups showed no difference (Fig. 2B), illustrating that IL-22 could help to ameliorate the hyperandrogenism in PCOS mice. In addition, the $\mathrm{LH}$ 
A

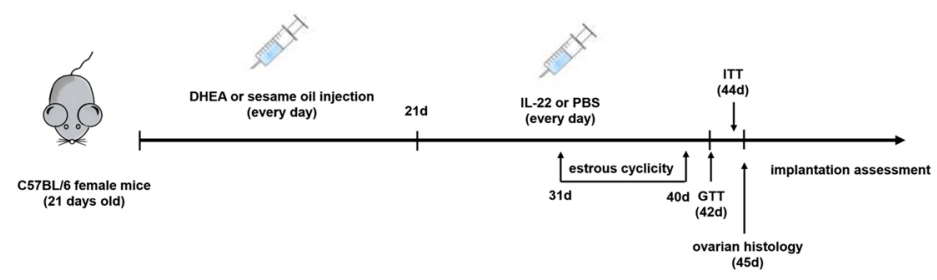

B

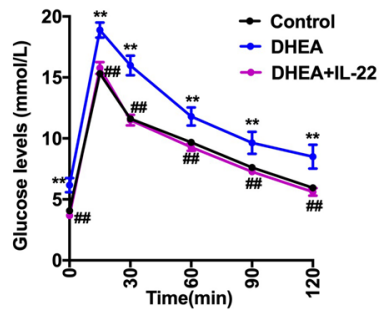

C

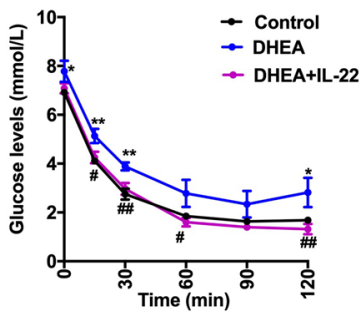

D

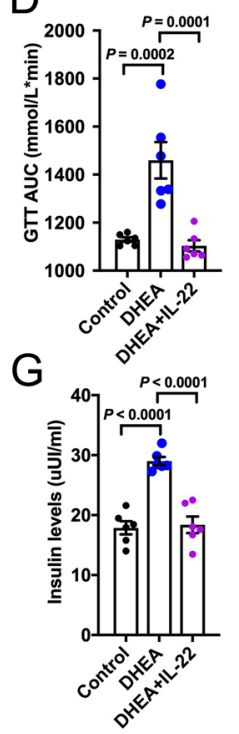

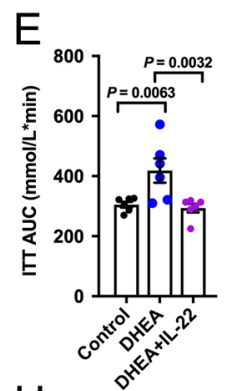

$\mathrm{H}$

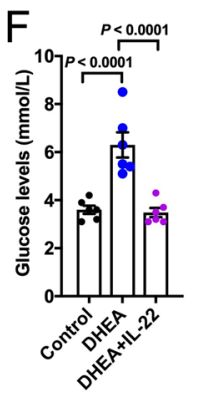

Figure 1

IL-22 treatment reverses metabolic dysfunction in PCOS mice. (A) Timeline for the control, DHEA, and DHEA + IL-22 mice. (B) The glucose tolerance test (GTT). (C) The insulin tolerance test (ITT). (D) The GTT AUC. (E) The ITT AUC. (F) Glucose levels. (G) Insulin levels. (H) HOMA-IR. $n=6$ per group. Data are plotted as the means \pm s.E.M. $* P<0.05$ represent DHEA vs control, $* * P<0.01$ represent DHEA vs control, $\# P<0.05$ represent DHEA + IL-22 vs DHEA, $\# \# P<0.01$ represent DHEA + IL-22 vs DHEA. One-way ANOVA with Tukey's multiple comparison post-hoc test was used to analyze the glucose levels of the three groups, where every group had six mice. Every point represented the glucose average level of every group, and error bar showed the s.E.M. of the glucose level. The differences between groups, as well as the difference between every two groups were analyzed. $P$ values were determined by one-way ANOVA with Tukey's multiple comparison post-hoc test. A full color version of this figure is available at https://doi.org/10.1530/JOE-19-0589.

levels also decreased with additional IL-22, while there was no difference in FSH levels. It is worth noting that the LH/FSH ratio, which is an important disease feature in patients with PCOS, was also decreased in the DHEA+IL-22 group compared with the ratio in the DHEA group (Fig. 2C, D and E). Taken together, exogenous IL-22 improved the disturbed hormone levels in PCOS by ameliorating the testosterone levels, LH levels, and the LH/FSH ratio.

\section{The ovarian dysfunction could be improved with additional IL-22}

Estrous cycles were assessed by daily examination of the vaginal smears to evaluate the reproductive function of the mice. As shown in the line graph, the DHEA mice had disrupted estrous cycles, characterized by a constant estrus stage. The control group and DHEA+IL-22 mice
A

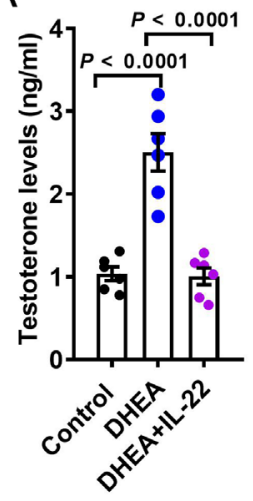

$\mathrm{B}$

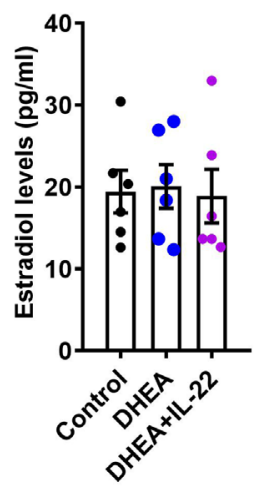

C

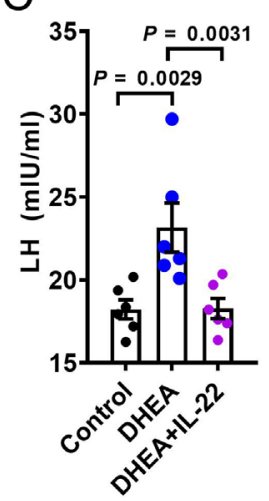

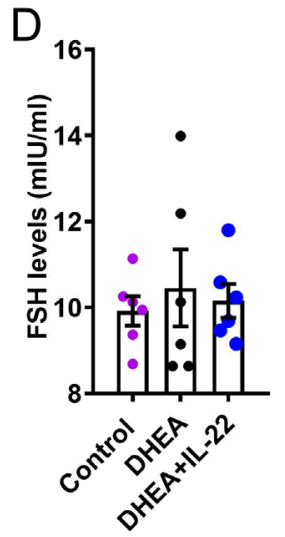

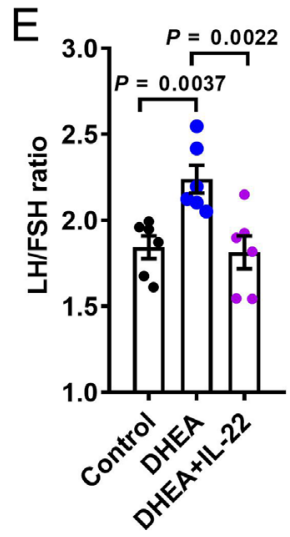

Figure 2

IL-22 treatment improved hormone disorders in PCOS mice. (A) Testosterone levels. (B) Estradiol levels. (C) LH levels. (D) FSH levels. (E) The LH/FSH levels. $n=6$ per group. Data are plotted as the means \pm S.E.M. $P$ values were determined by one-way ANOVA with Tukey's multiple comparison post-hoc test. A full color version of this figure is available at https://doi.org/10.1530/JOE-19-0589. 
A

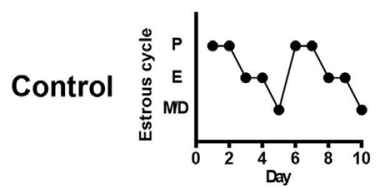

DHEA

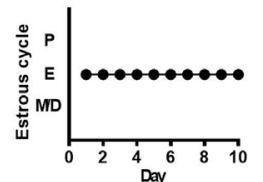

DHEA+IL-22

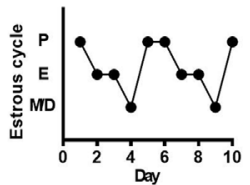

C

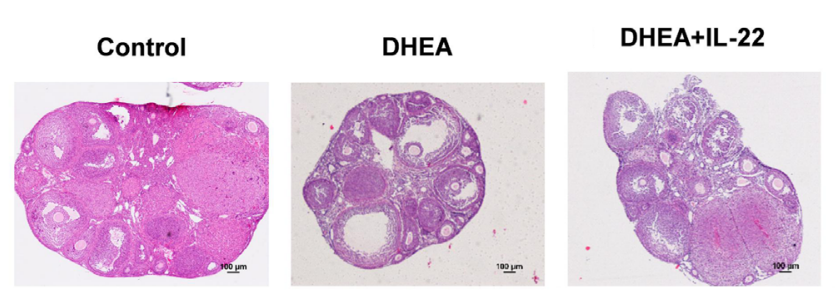

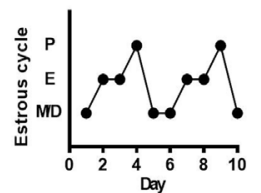
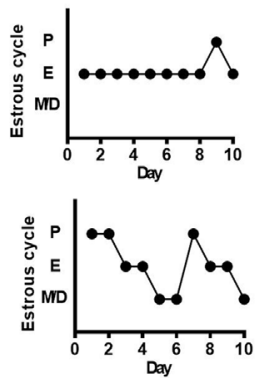
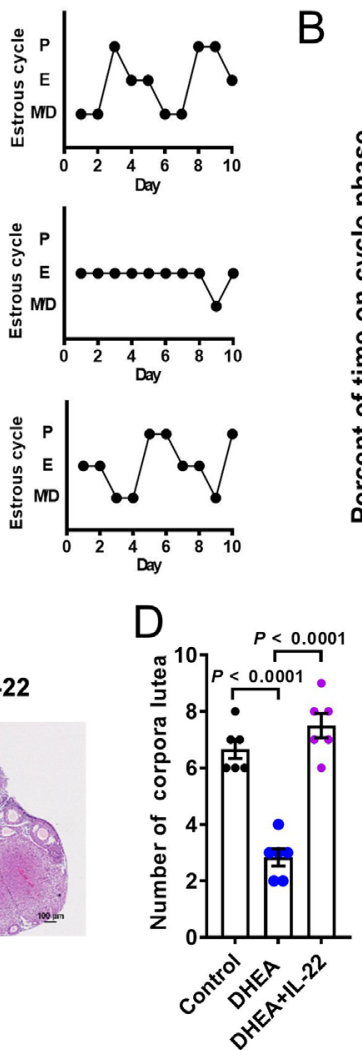

B

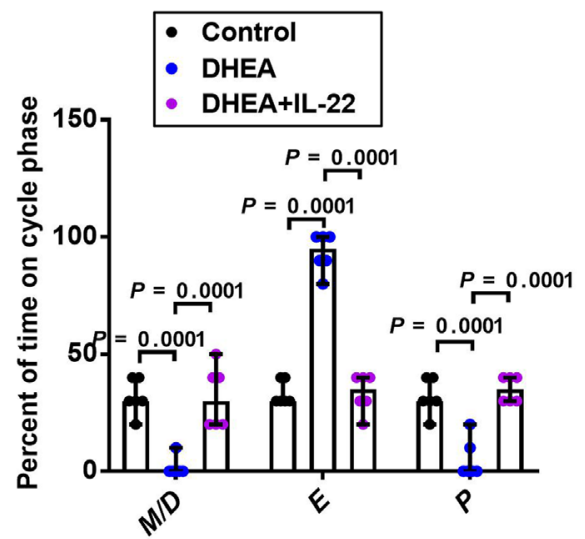

E

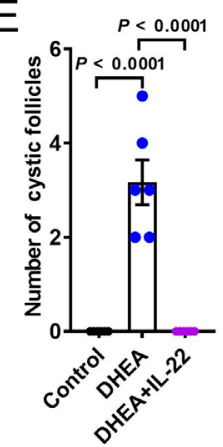

F

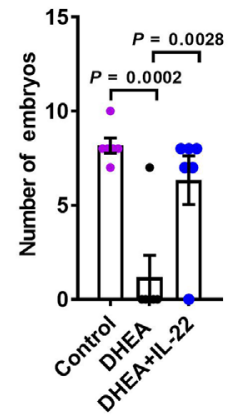

Figure 3

The ovary dysfunction could be improved with additional IL-22. (A) Representative estrous cycles of mice in the control, DHEA, and DHEA + IL-22 groups. P: preoestrus; E: estrous; D: diestrus. (B) The percent time in cycle phases of the three groups. (C) Hematoxylin-eosin (H\&E) staining of representative ovaries. Images are representative of three independent experiments with similar results. (D) The number of corpora lutea. (E) The number of cystic follicles. (F) The number of embryos. $n=6$ per group. For B, $P$ values were determined by Kruskal-Wallis test followed by Dunn's post-hoc test and data are presented as medians with interquartile ranges. $P$ values were determined by one-way ANOVA with Tukey's multiple comparison post-hoc test and data are presented as means \pm S.E.M. for D-F. A full color version of this figure is available at https://doi.org/10.1530/JOE-19-0589.

had a normal estrous cycle of 4 to 5 days (Fig. 3A). Quantitative analysis of the percentages of the estrous cycle in different stages also revealed that IL-22 treatment could improve the disrupted estrous cycle of DHEA mice to a regular estrous cycle (Fig. 3B). The ovary morphology of the DHEA group had typical PCOS changes, including a reduced number of corpora lutea and the appearance of cystic follicles (Fig. 3C, D and E). Under IL-22 treatment, the PCOS mice showed an increased number of corpora lutea and disappearance of cystic follicles to similar levels as shown in the control group (Fig. 3C, D and E). To further investigate the role of IL-22 administration on embryo formation or implantation in DHEA-induced PCOS mouse models, the embryo numbers were measured 10 days after vaginal plug formation. The number of embryos was significantly reduced in the DHEA group compared to control mice, while administration of IL-22 remarkably reversed the decline of embryo numbers under DHEA treatment (Fig. 3F). These results showed that IL-22 could help to improve ovarian functions, such as disrupted estrous cycles, abnormal ovary morphology, and the decreased number of embryos in DHEA mice.

\section{IL-22 treatment promotes white adipose tissue browning in PCOS mice}

White adipose tissue browning improves glucose metabolism and has a therapeutic role in PCOS (Qi et al. 2019). We weighed the mice every 2 days and there was no difference among the three groups. To further explore the possible mechanisms by which IL-22 regulates ovarian function and insulin resistance in androgen-induced PCOS mice, markers related to beiging, including $U c p 1, P g c 1 \alpha$, Cited1, Cox8b, Nr2f6, and Prdm16, were quantitatively measured in s.c. adipose tissues. The mRNAs of the brown adipose tissue markers $U c p 1, P g c 1 \alpha$, Cited 1 , and Cox $8 b$ were detected in the brown adipose tissues from each group. The relative Ucp1, Pgc1a, and Cited1 mRNA levels in s.c. 
A
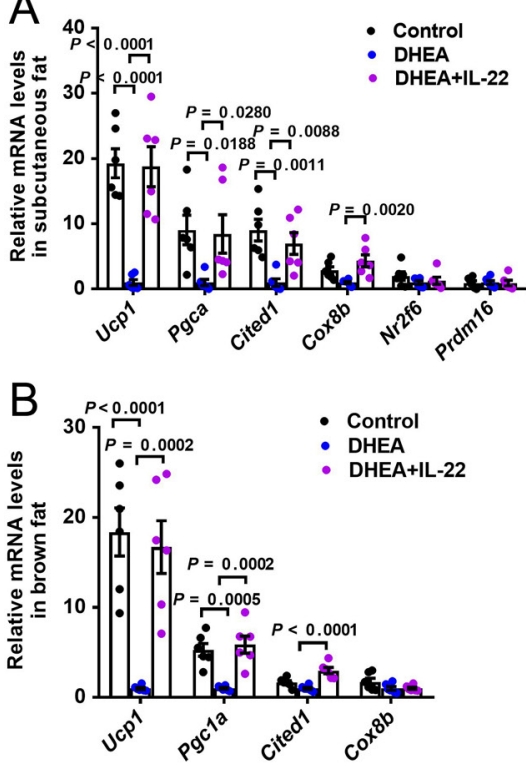

C

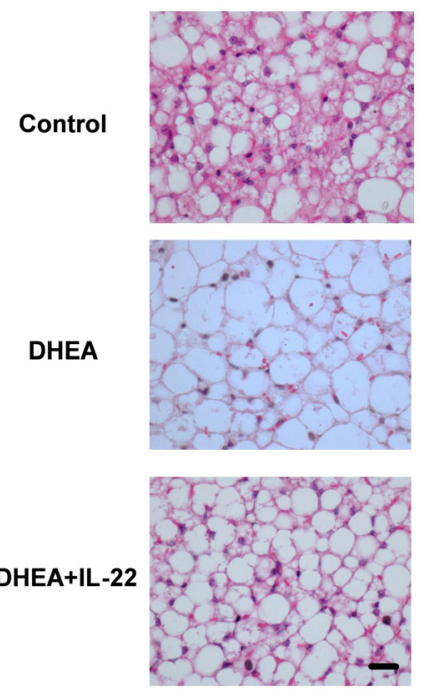

$\mathrm{D}$

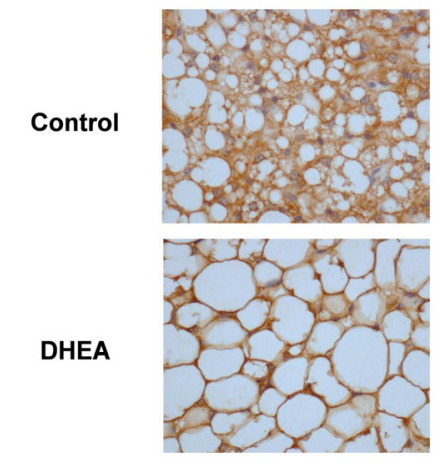

DHEA+IL-22

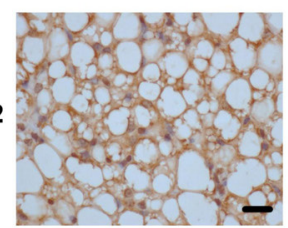

Figure 4

IL-22 treatment promotes white adipose tissue browning in PCOS mice. (A) Ucp1, Pgc1 $\alpha$, Cited1, Cox8b, Nr2f6, and Prdm16 mRNA expression measured by qPCR in subcutaneous fat. (B) Ucp1, Pgc1 $\alpha$, Cited1, and Cox $8 b$ mRNA expression measured by qPCR in brown fat tissue. (C) Hematoxylin-eosin staining of representative subcutaneous fat. Images are representative of three independent experiments with similar results, scale bars: $40 \mu \mathrm{m}$. (D) UCP-1 immunohistochemical staining of subcutaneous fat. Images are representative of three independent experiments with similar results, scale bars: $40 \mu \mathrm{m}$. Mice were exposed to $4^{\circ} \mathrm{C}$ cold for $12 \mathrm{~h}$ before the experiments. $n=6$ per group. Data are plotted as the means \pm S.E.M. $P$ values were determined by one-way ANOVA with Tukey's multiple comparison post-hoc test. A full color version of this figure is available at https://doi.org/10.1530/JOE-19-0589.

fat and Ucp1 and Pgc1a mRNA levels in brown fat of were significantly decreased in DHEA group compared with the control group (Fig. 4A and B). The DHEA+IL-22 group had significantly increased levels of Ucp1, Pgc1a, Cited1, and Cox8b mRNAs in s.c. fat (Fig. 4A) and Ucp1, Pgc1a, and Cited 1 mRNAs in brown fat compared to those of the DHEA group (Fig. 4B). S.c. fat underwent H\&E staining to show the adipose tissue morphology. IL-22 treatment was sufficient to induce significant browning of white adipose tissues, as revealed by the presence of many multilocular cells within the tissues compared with the DHEA group (Fig. 4C). Consistently, immunostaining analysis for UCP1 in s.c. fat tissues revealed that DHEA+IL-22 mice had higher levels of UCP1 (Fig. 4D). These findings support the view that IL-22 treatment promotes white adipose tissue browning in a DHEA-induced PCOS mouse model.

\section{Discussion}

Our previous study showed that IL-22 was decreased in PCOS patients. Transplantation of $B$. vulgatus to mice resulted in a PCOS-like phenotype and reduced interleukin-22 secretion. Giving IL-22 to $\mathrm{AMH}-$ or $B$. vulgatus-induced PCOS mice could help ameliorate the PCOS-like symptoms (Qi et al. 2019). Although testosterone levels were both increased in the AMH- or B. vulgatus-induced PCOS mice, these mice were not androgen-induced PCOS mice. Hyperandrogenism is the primary manifestation of PCOS. One etiologic hypothesis of PCOS is that exposure to excessive androgens early in life leads to PCOS in adulthood (Shi \& Vine 2012). DHEA is the first androgen to rise in the female peripubertal period (Mahesh \& Greenblatt 1962), and DHEA was used to induce PCOS in rats as early as 1962 (Roy et al. 1962). Therefore, DHEA-induced mice could better represent the hyperandrogenic phenotype in PCOS patients. To verify the role of IL-22 in androgen-induced PCOS, the DHEA model was used in the current study.

PCOS is a complex syndrome with endocrine and metabolic disorders. Environmental, genetic, and epigenetic factors are involved in the pathogenesis of PCOS. Gene mutations in 15 loci were associated with PCOS etiology, including the LHCGR (LH/CG receptor), THADA (thyroid adenoma associated), and DENNDIA (DENN domain-containing protein IA) genes (Filippou \& Homburg 2017). However, different definitions of PCOS contribute to the discrepancies in the genetic analyses in these studies. The ethnic and geographic heterogeneities are well known in PCOS patients. Hyperexposure of 
the fetus in utero to testosterone, hypomethylation of the $A M H$ gene, and telomere shortening are considered epigenetic changes in PCOS pathophysiology (Filippou $\&$ Homburg 2017). Heritable factors and acquired obesity were found to be involved in the pathogenesis of PCOSassociated insulin resistance (Rosenfield \& Ehrmann 2016). However, the existence of otherwise unexplained insulin resistance unrelated to genetics or diet/obesity makes the investigation of other influences an important area of research. IL-23, IL-6, IL-21, STAT3, Notch, and aryl hydrocarbon receptor (AhR) were reported to activate the expression of IL-22 (Lee et al. 2011). ROR $\gamma \mathrm{t} / \mathrm{ROR} \alpha$ and basic leucine zipper transcription factor (BATF) also control IL-22 production (Ouyang et al. 2011). The endogenous IL-22 inducer needs further investigation.

While there are a few PCOS mice models, we chose a common androgen-induced rodent model (PCOS-DHEA mice model) because the DHEA model is better suited to examine metabolic features and adiposity, as well as hormonal changes and ovary morphology. Another widely used PCOS model is the model of DHT injection during prenatal period. But the PCOS mice established by this method had low levels of androgen. As the current study focused on PCOS mice under high androgen state, it is not suitable to apply DHT model (Shi \& Vine 2012, Osuka et al. 2018). We aimed to investigate 'high androgen-induced' PCOS.

Typically, prepubertal rats, aged approximately 22 days, were injected daily with DHEA $(6 \mathrm{mg} / 100 \mathrm{~g}$ body weight, dissolved in $0.2 \mathrm{~mL}$ of sesame oil) for up to 20-27 days (Shi \& Vine 2012). In our previous papers, we also detected the estrous cycle, ovary morphology, and hormones levels to detect whether the phenotype is fully established compared with control. We found that after 21 days treatment, the DHEA group had disturbed estrous cycle, abnormal ovary morphology, and elevated testosterone and LH levels, these were the characteristics of DHEA-related hyperandrogenemia, anovulation, and cystic ovaries, and the mice also developed aberrations in insulin/glucose metabolism (Qi et al. 2017, 2019). These results demonstrate that the PCOS phenotype is fully established after 21 days of treatment of 3-week-old mice. We used IL-22 after 21 days of DHEA treatment, so it should be IL-22 that is truly reversing the abnormalities. We had also detected the preventative effect of IL-22 in our previous paper in a different model (Qi et al. 2019), when the IL-22 was given together with B.vulgatus for 21 days at 3 weeks old. While some studies showed that PCOS mice stayed at the diestrous (Yuan et al. 2016, Tata et al. 2018), the DHEA mice remained most of the time at estrous in our previously reported and current studies (Qi et al. 2019). Different PCOS animal models may display different estrus cycle stages due to different levels of androgens or LH, but the similarity is that these PCOS models all have a disturbed estrous cycle. This DHEA model had the infertility metabolic phenotypes similar to PCOS women (van Houten \& Visser 2014). We have insufficient evidence for the time point of embryo loss in this research. The implantation of mice occurs at about E4.5. We collected the samples at day 10 after vaginal plug formation to examine the embryo numbers. In our experiment design, we could not distinguish whether embryo loss occurs before or during implantation. In order to answer this question, we could take zygotes and culture them in vitro to examine the rate of blastocyst formation, because mice implant at the blastocyst stage, or we could take the uterus at E3.5 and flush the embryo. In the future, additional experiments are needed to give more evidence to uncover whether the embryo loss was at implantation or during pre-implantation development.

In our previous study, IL-22 levels were reduced in PCOS mice (Qi et al. 2019). This is consistent with the observations in obese mice, which indicate that IL-22 improves metabolic disorders in obese mice. The production of IL-22 is impaired in Citrobacter rodentiumchallenged obese mice. Interestingly, IL-22 receptordepleted mice tend to develop metabolic disorders when fed a high-fat diet. IL-22 administration to genetic or high-fat diet-induced obese mice improved metabolic symptoms, including insulin resistance (Wang et al. 2014). It was reported that IL-22 regulates metabolic syndrome in multiple ways, including downregulating the signal for food intake in the hypothalamus; reducing the levels of endotoxin, glucose, lipids, and LPS in the blood; enhancing lipolysis and fatty acid oxidation; preventing steatosis in the liver; enhancing mucosal immunity, resistance to infection, and gut integrity in the intestine; inhibiting apoptosis in islet beta cells; inducing STAT3 activation; and downregulating inflammation in adipose tissue (Dalmas \& Donath 2014, Wu \& Ballantyne 2014, Sabat \& Wolk 2015). The reduction of inflammatory factors and macrophage numbers by IL- 22 could be the reason why IL-22 improved insulin resistance. Based on the exogenous administration of IL-22, the current study revealed that IL-22 regulates insulin resistance through browning of white adipose tissue.

STAT3 acts as the crucial downstream molecule of the IL-22 receptor (Dudakov et al. 2015). It was reported that administration of IL-22 enhances the activation of STAT3 in adipose tissue (Wang et al. 2014). STAT3 promotes 
fat oxidation by activating its downstream molecule AMP kinase (Pedersen \& Febbraio 2008). In addition to lipid oxidation, AMPK acts as the center regulator to participate in the browning of white adipose tissue (Lopez et al. 2016). Further studies are needed to verify whether the STAT3-AMPK pathway mediates browning by IL-22.

Browning describes the beiging of adipocytes in white adipose tissue. Beige adipocytes have a similar function to brown adipose tissue, which can increase the metabolic rate and improve insulin resistance (Bartelt \& Heeren 2014, Brestoff \& Artis 2015). White adipose tissue browning increases the metabolic rate and improves insulin resistance (Fang et al. 2015). A recent study revealed that transplantation of brown adipose tissue reversed anovulation, hyperandrogenism, polycystic ovaries, insulin resistance, and infertility in DHEA-induced PCOS rats. These effects may be mediated by adiponectin (Yuan et al. 2016). The thermogenic markers in s.c. fat and brown fat were dramatically elevated after IL-22 administration in a PCOS-like mouse model, indicating that adipose tissue browning participated in the mechanisms of IL-22regulated improvements in insulin resistance and ovarian dysfunction associated with PCOS (Wang et al. 2014). This evidence provides new insights into the fat-ovary signal axis in metabolic diseases.

\section{Conclusions}

Infertility and metabolic syndrome are major complaints of women with PCOS. Owing to the limitations of understanding the mechanism, etiologic-specific treatments for infertility and insulin resistance are lacking in PCOS patients. Our study illuminates the therapeutic effect of IL-22 in high androgen-induced PCOS and indicates that IL-22 may be a valid treatment for PCOS patients with a hyperandrogenism phenotype.

\section{Supplementary materials}

This is linked to the online version of the paper at https://doi.org/10.1530/ JOE-19-0589.

\section{Declaration of interest}

The authors declare that there is no conflict of interest that could be perceived as prejudicing the impartiality of the research reported.

\section{Funding}

This work was supported by the National Key Research and Development Program of China (2018YFC1003900 and 2018YFC1003200), the National Natural Science Foundation of China (81521002 and 81730038), Clinical Medicine Plus X - Young Scholars Project of Peking University (PKU2018LCXQ13 and PKU2019LCXQ025), Research Units of Comprehensive Diagnosis and Treatment of Oocyte Maturation Arrest (2019-12M-5-001), Chinese Academy of Medical Sciences, and China Postdoctoral Science Foundation Grant (2019M660367).

\section{References}

Antunes LC, Elkfury JL, Jornada MN, Foletto KC \& Bertoluci MC 2016 Validation of HOMA-IR in a model of insulin-resistance induced by a high-fat diet in Wistar rats. Archives of Endocrinology and Metabolism 60 138-142. (https://doi.org/10.1590/2359-3997000000169)

Bartelt A \& Heeren J 2014 Adipose tissue browning and metabolic health. Nature Reviews: Endocrinology 10 24-36. (https://doi.org/10.1038/ nrendo.2013.204)

Brestoff JR \& Artis D 2015 Immune regulation of metabolic homeostasis in health and disease. Cell 161 146-160. (https://doi.org/10.1016/j. cell.2015.02.022)

Dalmas E \& Donath MY 2014 A role for interleukin-22 in the alleviation of metabolic syndrome. Nature Medicine 20 1379-1381. (https://doi. org/10.1038/nm.3748)

Dudakov JA, Hanash AM \& van den Brink MR 2015 Interleukin-22: immunobiology and pathology. Annual Review of Immunology 33 747-785. (https://doi.org/10.1146/annurev-immunol-032414-112123)

Dumesic DA, Oberfield SE, Stener-Victorin E, Marshall JC, Laven JS \& Legro RS 2015 Scientific statement on the diagnostic criteria, epidemiology, pathophysiology, and molecular genetics of polycystic ovary syndrome. Endocrine Reviews 36 487-525. (https://doi. org/10.1210/er.2015-1018)

Fang S, Suh JM, Reilly SM, Yu E, Osborn O, Lackey D, Yoshihara E, Perino A, Jacinto S, Lukasheva Y, et al. 2015 Intestinal FXR agonism promotes adipose tissue browning and reduces obesity and insulin resistance. Nature Medicine 21 159-165. (https://doi.org/10.1038/nm.3760)

Filippou P \& Homburg R 2017 Is foetal hyperexposure to androgens a cause of PCOS? Human Reproduction Update 23 421-432. (https://doi. org/10.1093/humupd/dmx013)

Lee JS, Cella M, McDonald KG, Garlanda C, Kennedy GD, Nukaya M, Mantovani A, Kopan R, Bradfield CA, Newberry RD, et al. 2011 AHR drives the development of gut ILC22 cells and postnatal lymphoid tissues via pathways dependent on and independent of Notch. Nature Immunology 13 144-151. (https://doi.org/10.1038/ni.2187)

Lopez M, Nogueiras R, Tena-Sempere M \& Dieguez C 2016 Hypothalamic AMPK: a canonical regulator of whole-body energy balance. Nature Reviews: Endocrinology 12 421-432. (https://doi.org/10.1038/ nrendo.2016.67)

Mahesh VB \& Greenblatt RB 1962 Isolation of dehydroepiandrosterone and 17alpha-hydroxy-delta5-pregenolone from the polycystic ovaries of the Stein-Leventhal syndrome. Journal of Clinical Endocrinology and Metabolism 22 441-448. (https://doi.org/10.1210/jcem-22-4-441)

Norman RJ, Dewailly D, Legro RS \& Hickey TE 2007 Polycystic ovary syndrome. Lancet 370 685-697. (https://doi.org/10.1016/S01406736(07)61345-2)

Osuka S, Nakanishi N, Murase T, Nakamura T, Goto M, Iwase A \& Kikkawa F 2018 Animal models of polycystic ovary syndrome: a review of hormone-induced rodent models focused on hypothalamuspituitary-ovary axis and neuropeptides. Reproductive Medicine and Biology 18 151-160. (https://doi.org/10.1002/rmb2.12262)

Ouyang W \& O'Garra A 2019 IL-10 family cytokines IL-10 and IL-22: from basic science to clinical translation. Immunity 50 871-891. (https://doi.org/10.1016/j.immuni.2019.03.020)

Ouyang W, Rutz S, Crellin NK, Valdez PA \& Hymowitz SG 2011 Regulation and functions of the IL-10 family of cytokines in inflammation and disease. Annual Review of Immunology 29 71-109. (https://doi.org/10.1146/annurev-immunol-031210-101312) 
Pedersen BK \& Febbraio MA 2008 Muscle as an endocrine organ: focus on muscle-derived interleukin-6. Physiological Reviews 88 1379-1406. (https://doi.org/10.1152/physrev.90100.2007)

Qi X, Zhang B, Zhao Y, Li R, Chang HM, Pang Y \& Qiao J 2017 Hyperhomocysteinemia promotes insulin resistance and adipose tissue inflammation in PCOS mice through modulating M2 macrophage polarization via estrogen suppression. Endocrinology 158 1181-1193. (https://doi.org/10.1210/en.2017-00039)

Qi X, Yun C, Sun L, Xia J, Wu Q, Wang Y, Wang L, Zhang Y, Liang X, Wang L, et al. 2019 Gut microbiota-bile acid-interleukin-22 axis orchestrates polycystic ovary syndrome. Nature Medicine $\mathbf{2 5}$ 1225-1233. (https://doi.org/10.1038/s41591-019-0509-0)

Rosenfield RL \& Ehrmann DA 2016 The pathogenesis of polycystic ovary syndrome (PCOS): the hypothesis of PCOS as functional ovarian hyperandrogenism revisited. Endocrine Reviews 37 467-520. (https:// doi.org/10.1210/er.2015-1104)

Roy S, Mahesh VB \& Greenblatt RB 1962 Effect of dehydroepiandrosterone and delta4-androstenedione on the reproductive organs of female rats: production of cystic changes in the ovary. Nature 196 42-43. (https://doi. org/10.1038/196042a0)

Rutz S, Wang X \& Ouyang W 2014 The IL-20 subfamily of cytokines from host defence to tissue homeostasis. Nature Reviews: Immunology 14 783-795. (https://doi.org/10.1038/nri3766)

Sabat R \& Wolk K 2015 Deciphering the role of interleukin-22 in metabolic alterations. Cell and Bioscience 5 68. (https://doi. org/10.1186/s13578-015-0060-8)
Shi D \& Vine DF 2012 Animal models of polycystic ovary syndrome: a focused review of rodent models in relationship to clinical phenotypes and cardiometabolic risk. Fertility and Sterility 98 185-193. (https://doi.org/10.1016/j.fertnstert.2012.04.006)

Tata B, Mimouni NEH, Barbotin AL, Malone SA, Loyens A, Pigny P, Dewailly D, Catteau-Jonard S, Sundstrom-Poromaa I, Piltonen TT, et al. 2018 Elevated prenatal anti-Mullerian hormone reprograms the fetus and induces polycystic ovary syndrome in adulthood. Nature Medicine 24 834-846. (https://doi.org/10.1038/s41591-018-0035-5)

van Houten EL \& Visser JA 2014 Mouse models to study polycystic ovary syndrome: a possible link between metabolism and ovarian function? Reproductive Biology 14 32-43. (https://doi.org/10.1016/j. repbio.2013.09.007)

Wang X, Ota N, Manzanillo P, Kates L, Zavala-Solorio J, Eidenschenk C, Zhang J, Lesch J, Lee WP, Ross J, et al. 2014 Interleukin-22 alleviates metabolic disorders and restores mucosal immunity in diabetes. Nature 514 237-241. (https://doi.org/10.1038/nature13564)

Wang X, Wong K, Ouyang W \& Rutz S 2019 Targeting IL-10 family cytokines for the treatment of human diseases. Cold Spring Harbor Perspectives in Biology 11 a028548. (https://doi.org/10.1101/cshperspect.a028548)

Wu H \& Ballantyne CM 2014 Inflammation versus host defense in obesity. Cell Metabolism 20 708-709. (https://doi.org/10.1016/j. cmet.2014.10.013)

Yuan X, Hu T, Zhao H, Huang Y, Ye R, Lin J, Zhang C, Zhang H, Wei G, Zhou H, et al. 2016 Brown adipose tissue transplantation ameliorates polycystic ovary syndrome. PNAS 113 2708-2713. (https://doi. org/10.1073/pnas.1523236113)

Received in final form 5 March 2020

Accepted 9 March 2020

Accepted Manuscript published online 9 March 2020 (c) 2020 Society for Endocrinology Published by Bioscientifica Ltd.
Printed in Great Britain 